\title{
Do we need B.C.G. vaccination?
}

\author{
V. H. SPRINGETT \\ M.D., F.R.C.P., F.F.C.M.
}

Birmingham Chest Clinic, 151 Great Charles Street, Queensway, Birmingham B3 3HX

THE DECISION to use, or to stop using, a method of immunization or vaccination depends on several factors; these include the epidemiological situation in the community concerned as well as evidence on the efficiency of the immunization procedure and the occurrence of complications. Rather less obviously, the availability of effective treatment for the condition to be prevented is also relevant.

Only the use of B.C.G. (Bacille Calmette-Guérin) vaccination in adolescents or young adults in England and Wales will be dealt with in this paper. For this group, it is reasonable to accept that the results of the Medical Research Council (MRC) trial (1972) are still valid, that is, about $75-80 \%$ protection of tuberculin-negative individuals over a period of approximately 15 years. Its continued effectiveness at this level is not just an assumption, for monitoring studies undertaken by Sutherland and the author (Springett, 1969; Springett and Sutherland, 1970; Sutherland and Springett, 1971) have shown no evidence of any falling off in effectiveness, and another recent study (British Thoracic and Tuberculosis Association (BTTA), 1975) has given confirmation of its continued effectiveness.

At this stage, two points must be stressed. Firstly, as long as there is any risk of tuberculous infection there is, as Waaler and Rouillon (1974) have pointed out, always an epidemiological gain from the continued use of B.C.G. vaccination. In strictly epidemiological terms the only counterbalancing effect would be from complications, and fortunately major complications from B.C.G. vaccination in adolescence are extremely rare if they occur at all. There is the problem of the scars, and sometimes slow healing, but anything more is quite exceptional. Secondly, the evidence of the MRC trial alone shows that B.C.G. should continue to be made available to young people entering risk situations including the hospital environment. The answer to the title of this paper is an unequivocal 'yes' for all hospital workers, for all tuberculin-negative contacts, and for all infants born into communities of immigrants who have been shown to have a high level of tuberculosis.

However, there is a question worth discussing, and to which it is not yet possible to give a firm answer when B.C.G. vaccination of all school-children at age 10-13 years is considered. The schools B.C.G. programme has been running for about 20 years, and it is common knowledge that opportunities for chance infection with tubercle bacilli have become much less in this period. Just how much the risks of tuberculous infections have fallen has recently been the subject of another study (Christie, 1974; Sutherland, 1974) which showed that in 1972 only $1.15 \%$ of 13-year-old children in Britain were tuberculinpositive. This corresponds with an annual risk of infection of less than 1/1,000. Evidence from this and other sources shows that the annual risk of infection is falling by $13 \%$ each year; this can be more conveniently expressed as a halving of the annualo risk of infection every 5 years.

B.C.G. vaccination protects mainly against tuberculosis occurring within a few years of primary? infection. If the number of primary infections is falling, then the number of cases of tuberculosis prevented by B.C.G. must also be falling, even though B.C.G. is maintaining its $80 \%$ efficiency$80 \%$ of zero is zero, and $80 \%$ of a very small number is also very small.

It was against this background that the Research Committee of the BTTA organized a study of the tuberculin and B.C.G. histories of all persons aged 15-19 years notified in England and Wales in 1973 (BTTA, 1975). About $75 \%$ of all notifications were reported to the co-ordinator, and adequate information about the status of these individuals in the schools B.C.G. scheme was obtained for the great majority. A considerable number had to be excluded as not eligible for the schools scheme, mainly because they had entered this country after the usual age for B.C.G. vaccination. The results for those eligible for the schools scheme showed a notification rate of $2 \cdot 72 / 100,000$ in those who had been given B.C.G. vaccination. The estimation of a notification rate for tuberculin negatives who were not vaccinated is a little complicated and involves some assumptions; the method is fully explained in the paper, and yields a rate of $12 \cdot 95 / 100,000$. Relating the rate of $2 \cdot 72 / 100,000$ observed in B.C.G. vaccinated subjects to the rate of $12 \cdot 95 / 100,000$ estimated for negative 
unvaccinated subjects shows $79 \%$ protection by B.C.G. vaccination.

When allowance is made for the under-reporting of notifications to this study, it is found that about fifteen notifications were prevented in persons aged 15-19 years in England and Wales in 1973 for each 100,000 vaccinations performed when they were at school.

The protective effect of B.C.G. vaccination is not confined to one year, nor to a limited age period, and some further calculations are required to estimate the number of notifications prevented over a 15-20 year period by 100,000 vaccinations in any given year. As well as allowing for this extended period of time for which protection lasts, the calculations have also to take into account the declining risk of infection, which, as already indicated, is halving every five years. The results of these calculations (BTTA, 1975) suggest that in the period centred on 1968 each 100,000 vaccinations prevented about 133 notifications over the next 15 years. Sutherland (1974) has suggested a rather higher figure, about 178.

Another, possibly more meaningful, way of expressing this result is as the number of vaccinations needed to prevent one notification over the 15 years following vaccination. This comes out at $\mathbf{7 5 0}$ vaccinations in 1968, and is as low as ninety-four if projected back to 1953 . This provides a useful crosscheck since the MRC/B.C.G. trial results can be expressed as about seventy vaccinations in 1950-52 to prevent one case of tuberculosis in 15 years.

Taking the estimates forward in time, it suggests that in $1973,1,500$ vaccinations were needed to prevent one notification, rising to 3,000 in 1978 , and 6,000 in 1983.

On the basis of figures of this type, it is possible to study the comparative costs of the B.C.G. vaccination scheme and of the treatment of the cases it prevents. Such a study is in progress, but the results are not yet known*. One can assume that we are somewhere near the break-even point where there is little difference between the cost of the vaccination scheme and the cost of treating the cases it prevents.

This is far from saying that as soon as treating the cases is cheaper than the vaccination programme we should stop the vaccination programme. The prevention of the occurrence of tuberculosis is of greater importance to the individual than being treated for tuberculosis, no matter how effective the treatment. If there was no chemotherapy for tuberculosis, there could be no justification for stopping B.C.G. vaccination until there was no risk of infection at all. The usefulness of the comparative cost approach is to make reasonably sure that we continue the schools B.C.G. scheme at least as long as it is less expensive

* Now published: Stilwell, 1976. than treating the cases. It is the opinion of the author that we should continue well beyond this point, say by 5 or 10 years.

This was approximately the stage of the author's thinking a few months ago, it was then thought that it would be reasonable to give up the schools vaccination scheme in the not very distant future. However, the results of the BTTA study suggest that the termination of the schools B.C.G. scheme should be postponed.

The starting point for this new approach was to make an estimate of the number of notifications prevented in England and Wales in 1973 by the 20 years of the vaccination programme. Again, a number of assumptions have to be made and, depending on these, it is possible to come up with rather different answers. A round figure of 1,000 notifications prevented in 1973 is probably not too far from the truth-it could be a little less, it could perhaps be as high as 2,000 , but it is unlikely to be higher than that.

Now, 1,000 notifications is about $10 \%$ of all notifications in all age-groups in 1973 and represents a substantially greater benefit from the 20-year investment in the B.C.G. programme than was previously thought. It is a contribution towards tuberculosis control that is not to be lightly discarded. Since the protective effect of B.C.G. lasts 15-20 years, the full effect of stopping a B.C.G. programme will also take 20 years.

Calculation of the probable results of stopping the B.C.G. programme at various periods of time is complicated because of the falling infection risk and the duration of protective effect of B.C.G. In Table 1

TABLE 1. Estimates of additional notifications per year from stopping the schools B.C.G. programme at various times

\begin{tabular}{ccrrrrr}
\hline & \multicolumn{6}{c}{$\begin{array}{c}\text { Additional annual notifications that could } \\
\text { be prevented by continuing B.C.G. }\end{array}$} \\
\cline { 2 - 7 } \begin{tabular}{c} 
If B.C.G. Scheme $\begin{array}{c}\text { B. } \\
\text { stopped in: }\end{array}$ \\
\cline { 2 - 7 }
\end{tabular} & 1973 & 1978 & 1983 & 1988 & 1993 & 1998 \\
\hline 1973 & 0 & 125 & 125 & 94 & 62 & 31 \\
1978 & 0 & 0 & 62 & 62 & 47 & 31 \\
1983 & 0 & 0 & 0 & 31 & 31 & 15 \\
1988 & 0 & 0 & 0 & 0 & 15 & 15 \\
\hline
\end{tabular}

the additional notifications that would occur at 5year intervals if the schools B.C.G. programme were stopped at any of the stated years have been calculated. The starting point of the calculation is 1,000 cases prevented in 1973 , and a halving of the annual infection risk every 5 years. It is also assumed that there is no marked age-effect between adolescence and the early thirties, and that B.C.G. is effective over 20 years.

These calculations suggest that stopping the B.C.G. programme in 1973 would have meant more than 100 
extra notifications each year from 1978 to about 1990. Stopping in 1978 would mean accepting an additional sixty cases per year from about 1980 to 1990 , falling gradually to thirty cases per year at the end of the century. Whatever the results of the cost comparison, this represents an unacceptably large number of notifications that could be prevented.

On present evidence, stopping the schools B.C.G. scheme in 1983 would result in an additional thirty cases a year for several years and, as the author's view only, at about this level or below does it become reasonable to consider stopping the schools B.C.G. programme.

As indicated earlier, it is not yet possible to give an unequivocal answer to the question that is the title of this paper. Thus it can be concluded that:

(1) There is good reason to believe that the $80 \%$ protection over 15 years shown by B.C.G. vaccination in the MRC trial continues to be valid for adolescents in this country.

(2) This being so, no adolescent or young adult should be allowed to enter a risk situation unless known to be tuberculin positive or to have had an effective vaccination with B.C.G. This includes all hospital and laboratory workers.

(3) In a community with a rapidly declining risk of tuberculous infection a situation can be reached where the continued use of B.C.G. on a community scale may no longer be justified.

In epidemiological terms, there is an advantage from using B.C.G. as long as there is any risk of infection-because of the virtual absence of complications of B.C.G. vaccination.

In economic terms, there is a point where treating the few extra cases would be less costly than the B.C.G. programme. The only usefulness of this approach is to warn against too early cessation of the B.C.G. programme.
In more general terms, and purely subjectively, there is for the author a level of 'additional cases per year' from stopping the B.C.G. programme which is unacceptable-and this leads to the view that we probably do need our B.C.G. programme for another 10 years at least, with some further studies meanwhile to check whether these forecasts are being fulfilled.

\section{References}

British Thoracic and Tuberculosis Association (1975) Present effectiveness of B.C.G. vaccination in England and Wales. Tubercle, 56, 129.

Christie, P. (1974) National Tuberculin Survey (summary). British Thoracic and Tuberculosis Association Review, 4, 45.

Medical Research Council (1972) B.C.G. and vole bacillus vaccines in the prevention of tuberculosis in adolescence and early adult life. Fourth report to the Medical Research Council by its Tuberculosis Vaccines Clinical Trials Committee. Bulletin of the World Health Organization, 46, 371.

SPRINGETT, V.H. (1969) B.C.G. vaccination in Birmingham: An assessment of its contribution to tuberculosis control in 1956-67. Tubercle, 50, 159.

SPRINGetT, V.H. \& Sutherland, I. (1970) Comparison of the efficacy of liquid and freeze-dried strains of B.C.G. vaccine in preventing tuberculosis. British Medical Journal, 4, 148.

Stilwell, J.A. (1976) The benefits and costs of the Schools B.C.G. Vaccination Programme. British Medical Journal, $1,1002$.

Sutherland, I. \& SPRingett, V.H. (1971) A comparison of the protective efficacy of a liquid (Copenhagen) and a을 freeze-dried (Glaxo) strain of B.C.G. vaccine. International. Symposium on B.C.G. Vaccine, Frankfurt (Main) 1970; Symposium Series Immunobiological Standardization, 17, 49.

Sutherland, I. (1974) Future policy for tuberculosis control in Britain (summary). British Thoracic and Tuberculosis Association Review, 4, 46.

WAALER, H. \& Rouillon, A. (1974) B.C.G. vaccination policies according to the epidemiological situation. Bulletin of the International Union against Tuberculosis, 49, No. 1, 166. 